\title{
Membrane Potential and Response Changes in Mammalian Cochlear Hair Cells during Intracellular Recording ${ }^{1}$
}

\author{
PETER DALLOS \\ Auditory Physiology Laboratory (Audiology) and Department of Neurobiology and Physiology, Northwestern University, Evanston, \\ IIIinois 60201
}

\begin{abstract}
In the preceding paper (Dallos, P. (1985) J. Neurosci. 5: 1591-1608) some fundamental properties of cochlear inner and outer hair cells were considered. On the basis of intracellular recording from the low frequency region of the guinea pig cochlea, such measures as membrane potentials, the dependence of $A C$ and $D C$ receptor potential magnitude on stimulus level and frequency, and tuning characteristics relative to gross responses were discussed. During prolonged recording various changes in membrane and receptor potentials were observed. In the present paper the relationship between these variations is described and evaluated in the context of a simple hair cell model.
\end{abstract}

During intracellular recordings from hair cells in the acousticolateralis system the general aim is to determine response properties and, thus, to derive the cells' operating characteristics. In general, repeated measures are not obtained during a prolonged intracellular encounter and, consequently, no attention has been paid to possible changes over time. In this laboratory's recordings from the low frequency regions of the guinca pig cochlea, it was noted that both membrane potential and response variations were frequently seen. In this paper such variations are described and the relationships between the two indices of cell function are examined.

A great deal of information is now available on the electrical behavior of high frequency cochlear hair cells (Russell and Sellick, 1978, 1983; Nuttall et al., 1981; Goodman et al., 1982; Russell, 1983), of turtle cochlear hair cells (Fettiplace and Crawford, 1978; Crawford and Fettiplace, 1981), alligator lizard cochlear hair cells (Weiss, 1984), frog saccular hair cells (Hudspeth and Corey, 1977; Hudspeth, 1983), and lateral line hair cells (Harris et al., 1970; Flock et al., 1973). In the preceding paper (Dallos, 1985) material was presented on the general behavior of low frequency inner hair cells ( $\mathrm{HCs}$ ) as well as outer hair cells (OHCs). The present paper supplements those findings and focuses on labilities in responses and membrane potentials that can be observed during maintained contact with a single hair cell.

\section{Materials and Methods \\ A detailed description of experimental methods is given by Dallos (1985); consequently, only a few salient items are mentioned here.}

Received September 11, 1984. Revised December 10, 1984; Accepted December 11, 1984

${ }^{1}$ Technical assistance was rendered by Fvan Relkin, Jamie Begelman, and William Lutz. Joseph Santos-Sacchi and Elizabeth Oesterle took part in various phases of data collection. Research was supported by National institute of Neurological and Communicative Disorders and Stroke Grant NSO8635.
Preparation. All recordings were obtained from young albino guinea pigs. The animals were anesthetized, tracheotomized, and artificially rcspirated after the administration of Flaxedil. A ventrolateral approach was used to the auditory bulla, which was opened, and the bony cochlea was transilluminated. To create a path for the recording electrode, a fenestra was prepared through the cochlear bone over the desired recording location. This was either the third or the fourth turn. The window was placed over the spiral ligament covering of the stria vascularis. The shadow of the organ of Corti could be seen through the opening and this facilitated the aiming of the electrodes.

Stimuli. Either series of tone burst stimuli or pseudorandom noise stimuli were used. These were generated under computer control (PDP-11/34), and the responses to them were stored in computer memory for off-line data analysis. Repeated measures to the same stimulus during a given cell penetration were obtained various ways. The pseudorandom noise sequences were presented at five sound levels with $10 \mathrm{~dB}$ spacing. Once the sequence was concluded, another was delivered-but at five different levels. One or two levels were common between the two; thus, one measure of change could be derived. During very prolonged recordings from a given cell, the noise sequences were delivered several times, so that opportunities for multiple comparisons were available. Tone burst sequences were also presented in a repeated fashion, either to obtain multiple measures or to generate more sound levels (a given sequence delivered five sound levels). In later experiments the tone burst series were so arranged that the first frequency to be presented (usually at the anticipated best frequency (BF)) was repeated at the end of the entire sequence. Since the presentation of a complete tone burst array consumed about $4.6 \mathrm{~min}$, this repeated presentation provided an excellent measure of response stability.

In several figures of this paper membrane potentials are shown as a function of time. Attached to the traces are time marks indicating the duration and occurrence of response measurements that are used for comparisons. In the figures the comparisons are based on the $\mathrm{AC}$ receptor potential magnitude at the BF. These are derived either from low level tone burst data or from the appropriate frequency component in the response sepctrum obtained with low level noise inputs. Low signal levels are used in these comparisons to avoid, as much as possible, the complicating influence of nonlinear processes that are pronounced at high sound levels, especially around the $\mathrm{BF}$.

Recording. Microelectrodes fabricated from $1-\mathrm{mm}$ glass capillaries were used. Their resistances ranged from 80 to 200 megohms when filled with 3 $\mathrm{M} \mathrm{KCl}$. DC potentials and responses were recorded with a high impedance preamplifier (Mentor, type N950), further amplified and displayed on a chart recorder as well as stored in computer memory. Off-line data evaluation consisted of Fourier analysis of averaged, stored waveforms of responses to pseudorandom noise sequences or tone bursts. AC response components are obtained from either method as the fundamental component in the response to a given tone burst, or as a spectral component in the response noise spectrum. DC responses are derived from plots of the averaged response to tone bursts, by measuring the response asymmetry. In other words, the DC response is taken as the difference between the base line and the midpoint between the positive and negalive response peaks.

\section{Results}

Although stable recordings are frequently seen, the membrane potentials of cochlear hair cells tend to change during maintained contact. Such changes would be considered uninteresting byproducts of differing quality recordings, were it not for the observation 
that unexpected receptor potential variations may accompany the membrane potenlial changes. To consider the covariation of the lwo electrical phenomena, a classification scheme is introduced. Using the membrane potential achieved at initial penetration of a cell as the reference, one can distinguish cases of steady, increasing (hyperpolarizing), and decreasing (depolarizing) "resting" potential epochs. In comparison with the direction of change in the membrane potential, we use the same classification of steady, increasing, or decreasing, for describing the change in receptor potentials. For simplicity, only one measure is used, the magnitude of the AC receptor potential, measured at the cell's BF at relatively low sound pressure levels so that a quasilinear measure may be utilized. Some other features of receptor potential changes, such as variations in tuning, and the behavior of the DC receptor potential are considered later. There are nine possible combinations of membrane and receptor potential changes when the simplified, three-way classification scheme is used. Of these nine possibilities, six were observed. They are described below.

The ideal recording situation is depicted in Figure 1a. Here the membrane potential is steady during an extensive recording period, and it is accompanied by an essentially unchanging response to sound. The companion case is shown in Figure $1 b$, where steady membrane potential is measured over time, in this case quite prolonged, but the response magnitude shows a relatively modest decline. A third possible case, that of steady membrane potential and increasing response, was not seen.

The situation one would expect, a priori, during intracellular recording is declining membrane potential accompanied by decreasing response. In fact, such cases are often seen. An example is given in Figure $2 a$, where the $\mathrm{IHC}$ membrane potential drops from its initial $-36 \mathrm{mV}$ value to about $-10 \mathrm{mV}$ by the second measurement of responses. $\mathrm{A} 9-\mathrm{dB}$ drop in the $\mathrm{AC}$ receptor potential is seen between the time periods. Not infrequently, the membrane potential disappears during recording. The stimulus-related potential drops to the level of the extracellular response in such situations.

A more surprising outcome of relating response magnitudes to

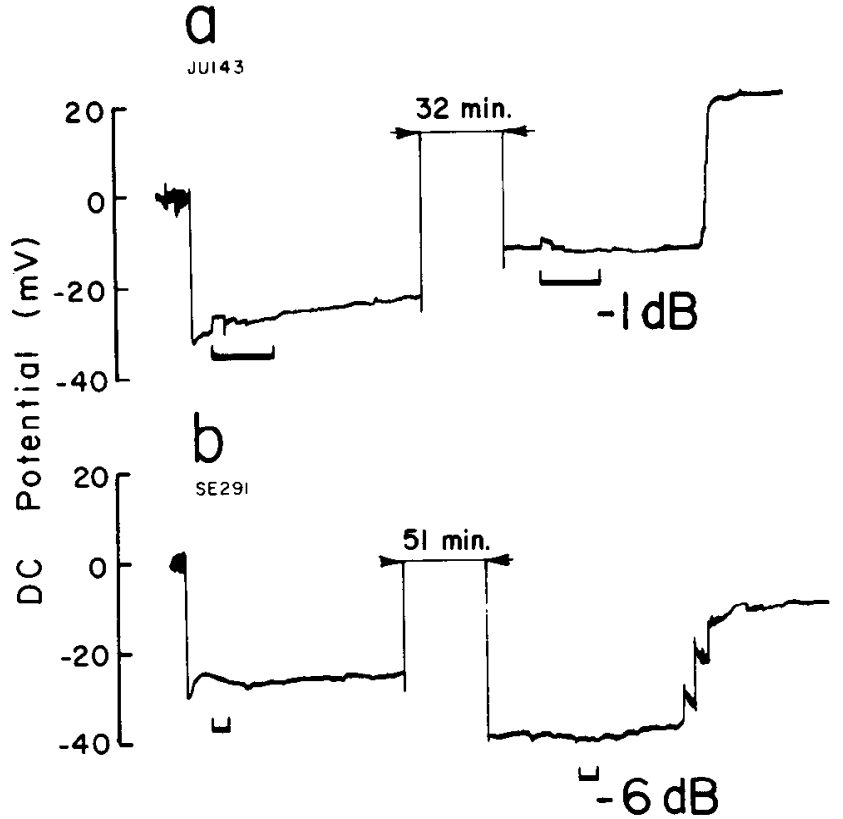

Figure 1. Two IHCs in which steady membrane potential was maintained during the length of the recording. $a$, Essentially constant receptor potential recorded throughout; $b$, receptor potential decreases during the prolonged recording. Horizontal bars indicate the position in time when the receptor potentials were obtained. Change in receptor potential from first to second measurement is indicated in decibels. Note that during recording the base line shifts somewhat. Nevertheless, when compared to extracellular space, the membrane potential at entry and exit is the same.

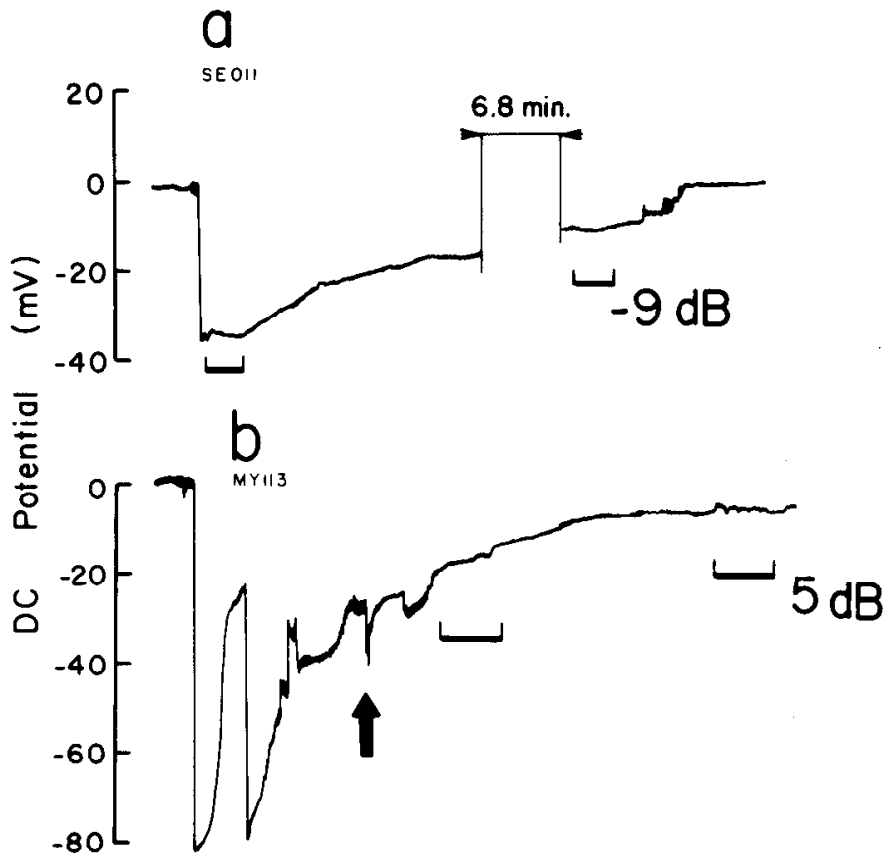

Figure 2. Two $\mathrm{HCS}$ in which the membrane potential declined during recording. In $a$, receptor potential decreases $9 \mathrm{~dB}$, whereas in $b$, it is increased $5 \mathrm{~dB}$. The heavy arrow in $b$ indicates the instant of acquiring the $\mathrm{IHC}$. Other details are as in Figure 1.

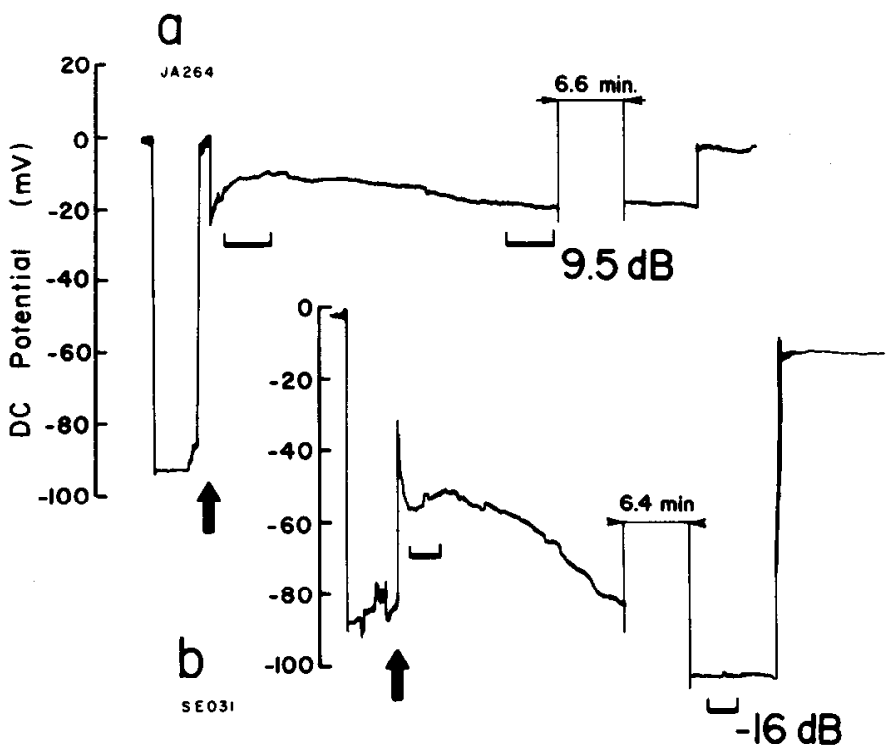

Figure 3. Recording from two hair cells in which the membrane potential increased during contact with the cells. $a$, Receptor potential increased 9.5 $\mathrm{dB}$ in this $\mathrm{IHC}$. $b$, The stimulus-related response decreased $16 \mathrm{~dB}$ in this $\mathrm{OHC}$. Arrows indicate the instant of penetration into hair cells. Other details are as in Figure 1.

membrane potentials is depicted in Figure $2 b$. Here the membrane potential declines from about $-40 \mathrm{mV}$ to less than $-10 \mathrm{mV}$ in about $3 \mathrm{~min}$, but during the drop a distinct increase in the response, +5 $\mathrm{dB}$ in this case, is noted. Examples for such inverse alterations are seldom seen but they present interesting cases for the consideration of possible mechanisms. There are no examples among the present samples for the situation in which the response remains constant while the membrane potential declines.

The final pair of examples describes the increase in membrane potentials after initial penetration of the cells. In Fig. 3a, during the approximately 3 min between measurements, the membrane poten- 
tial moves from about -10 to $-20 \mathrm{mV}$, along with a 9.5-dB increase in the response. This relationship, a counterpart of what has been seen in the previous category of declining membrane and receptor potentials, is an expected one. In other words, one intuitively seeks improved response with increasing membrane potential and vice versa. In Figure $3 b$ the unexpected is shown. The membrane potential of this $\mathrm{OHC}$ increases from -58 to $-95 \mathrm{mV}$, whereas the response drops $16 \mathrm{~dB}$. This intriguing pattern of behavior was seen in several hair cells. The course of this membrane potential change is approximately exponential. The time constant of the process has been estimated for five cells that exhibited this behavior. These ranged from 24 to $92 \mathrm{sec}$, with a median of $50 \mathrm{sec}^{2}$

In some situations membrane potential changes are accompanied by alterations in the frequency response characteristics of the cell as revealed by magnitude and phase data. Such tuning changes, however, do not necessarily occur whenever the membrane potential varies. As a matter of fact, it is possible to encounter distinct response magnitude changes accompanying membrane potential variations without a tuning change. To organize these various experimental results, let us return to Figures 1 to 3 . In Figure 1a the "normal" case is shown, in which stable membrane and receptor potentials are recorded over time. In all such cases the tuning remains invariant as well. In other words, the filter characteristics of the cells (discussed by Dallos, 1984, 1985, in detail) remain unaltered. Figure $1 b$ shows the case of stable membrane potential and declining response. There are several examples of this sort of pattern, all showing a lack of change in tuning. Thus, in cells for which membrane potential remains stable, the magnitude of the AC receptor potential may decline over time, but this occurs to the same degree at any frequency. Yet, stable membrane potential does not assure stable frequency response characteristics. In special cases where initial membrane potential alterations are followed by a stable period, one may see radical changes in tuning. For example, in Figure 4 the membrane potential first increases and then remains stable at a high value. The cell's tuning changes throughout the initial membrane potential increase. At the very end of the penetration, without changing membrane potential, the tuning is radically altered along with further decrease in response magnitude. Magnitude and phase data are presented in reference to organ of Corti response as seen initially, at the time when the membrane potential stabilized, and then again before stepping out of the cell. It is clear that the hair cell response, when referred to the gross potential in the adjacent fluid space, appears to be low-pass filtered (this matter is discussed in Dallos, 1985). With the passage of time the filter bandwidth increases and the magnitude of the response decreases. Then, without a change in membrane potential, at the end of the recording, the intracellular response is almost indistinguishable from the extracellular one, both in its magnitude and in its phase.

In Figures $2 a$ and $3 a$ cases of covariation between membrane and $A C$ receptor potential magnitude were shown. As a rule, in these situations the tuning remains quite stable. In the examples illustrated, no change in tuning occurs in spite of the increase or decrease in both membrane and receptor potential. There are cases in which the membrane potential disappears entirely over time without a clearcut transition from intracellular recording to extracellular. In such situations, of course, the filter properties of the cell are also altercd along with the response magnitude, and they assume extracellular character when a membrane potential is no longer discernible.

We have already seen one of the possibilities for opposing movements of membrane and receptor potentials. Figures 3 and 4 served as examples. The complementary case is depicted in Figure

\footnotetext{
${ }^{2}$ These changes, as well as others described elsewhere, cannot be attributed to alterations in electrode characteristics, such as "plugging." Electrode resistance is monitored during recordings and I am confident that the phenomena described herein have physiological origins.
}

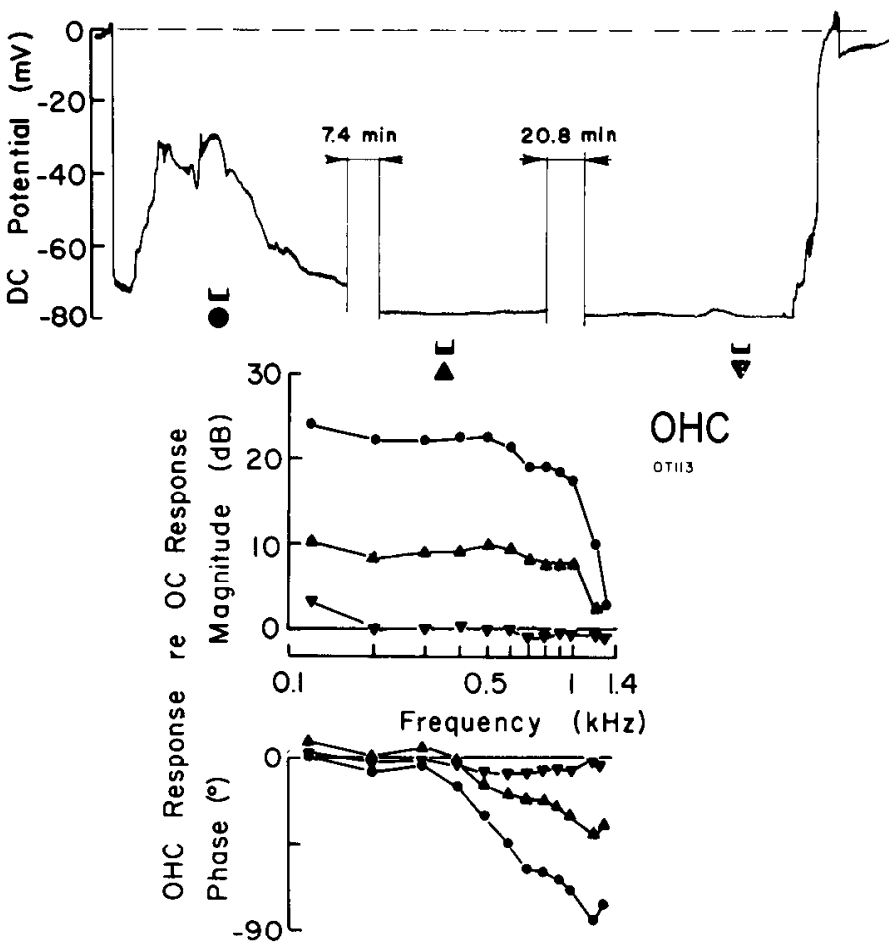

Figure 4. Time history of recording from a particular OHC. Top, Membrane potential versus time. Bottom. Frequency response (magnitude and phase) of $\mathrm{OHC}$ receptor potential referenced to the response seen in the organ of Corti fluid space $(O C)$ outside the hair cell. Symbols are keyed to correspond to different moments during recording when the intracellular responses were measured. Note that at the end of the recording $(\boldsymbol{\nabla})$ there is virtually no difference between intra- and extracellular measures of the AC response, in spite of the very large membrane potential.

$2 b$, in which the membrane potential declines whereas the $A C$ response increases. We have no $\mathrm{OHC}$ example for this situation, and very few $\mathrm{HCS}$. In $\mathrm{HHCs}$ this pattern is accompanied by a relatively complex change in the cell's filter characteristic. In such cases the increase in magnitude is prominent at low frequencies but gradually diminishes with increasing frequency. Low frequency phase changes are minimal, but as frequency increases, the phase lead is reduced. The ramifications of these changes are considered later.

In discussing lability of the recorded responses, some attention needs to be paid to the $\mathrm{DC}$ component of the receptor potential. Inasmuch as the DC response is a consequence of the cell's nonlinear behavior, which is not considered in this paper in any detail, only some cursory observations are made. It is our finding that the DC component is much more variable, from cell to cell and from instant to instant within the same cell, than the fundamental component of the $\mathrm{AC}$ response. Thus, the variability of $\mathrm{DC}$ response magnitude and frequency dependence is much greater among preparations than that of the corresponding $\mathrm{AC}$ characteristics. Similarly, there is a trend for the DC responses to be altered more radically during prolonged contact with a given cell. To show the astonishing degree of lability that one may see in the DC component, one example, our most radical one, will suffice. In Figure 5 we see a situation in which both membrane potential and the low level AC response at BF are essentially invariant, as is the endocochlear potential. There is, however, a starlling qualilalive change in the DC component of the receptor potential, accompanied by an increase in the high level $A C$ response. The polarity of the DC response is changed from the normal positive to the seldom-seen negative. Although this is the only example in our material where such bizarre changes occur, it is presented to demonstrate the possibility of a major alteration in the cell's nonlinear mechanism without a concomitant change in the membrane potential or the fundamental component of the $\mathrm{AC}$ response. More frequent occurrences are the simple 


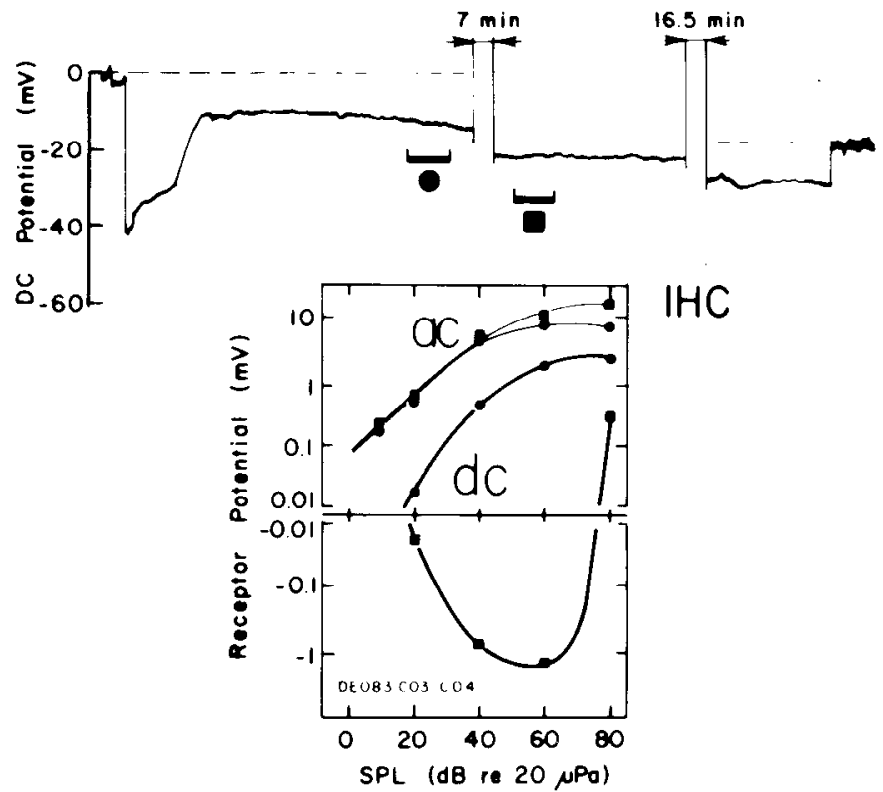

Figure 5. Time history of recording from a particular IHC. Top, Membrane potential versus time. Bottom, $\mathrm{AC}$ and $\mathrm{DC}$ input-output functions from the cell measured at $\mathrm{BF}$ at the two indicated time periods. Observe that during the first recording both $A C$ and $D C$ responses exhibit their expected pattern. The AC magnitude function bends over at high levels; the DC potentials are always positive. Seven minutes later the $A C$ plot is linearized somewhat (does not bend over), whereas the DC potential is now negative. These changes occur without alteration in the cell's membrane potential. Note that the base line shifts during the recording. This is the only figure in this paper which is based on data from a fourth turn hair cell.
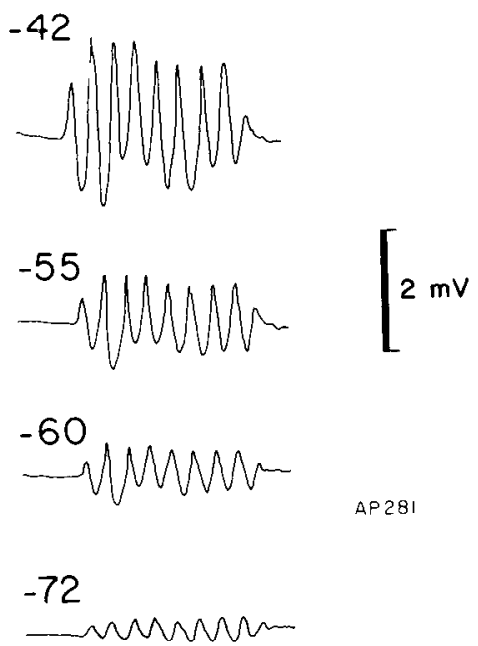

Figure 6. Averaged responses recorded at the same sound level from a given $\mathrm{OHC}$ whose membrane potential increased over time (pattern similar to that shown in Fig. $3 b$ ). The linearization of the response, i.e., decrease in peaked waveform and DC potential, is apparent as the membrane potential grows. The linearization may be a byproduct of the decrease in the response magnitude.

decline in the DC response much in excess of the change seen in the $\mathrm{AC}$ component.

The large increase in membrane potential and the concomitant decrease in response (Figs. $3 b$ and 4 ) is also accompanied by a process of linearization. A demonstration of this change is given in Figure 6 . The averaged response traces were obtained from an $\mathrm{OHC}$ as its membrane potential changed from an initial value of -42 to a steady-state $-72 \mathrm{mV}$ within a 2 -min period. As the traces indicate, the $\mathrm{AC}$ response decreased some $17 \mathrm{~dB}$ as the membrane potential increased and at the same time the response asymmetry disappeared.

\section{Discussion}

The only studies that have examined variations over time in hair cell membrane and receptor potentials are those of Brown et al. (1983) and Russell and Ashmore (1983). In both studies transient asphyxia or anoxia was induced and concomitant changes in $\mathrm{HC}$ membrane and receptor potentials were noted. General features were more severe changes in DC than in $A C$ receptor potentials (linearization of response) accompanied by hyperpolarization of the cell, and a deterioration of frequency selectivity. Anoxia produces a generalized effect on the state of the cochlea, including influences over both mechanical and electrophysiological processes. Consequently, it is difficult to parcel out the influence of this manipulation on the hair cell itself. In the material described here, the only affected entity is the hair cell from which the recording is made. Thus, a comparison between the present results and those derived from studies with induced anoxia is difficult to make.

Resting potentials. If the membrane potential of either type of hair cell changes in time after electrode penetration, then it is reasonable to assume that the variations are related to the presence of the foreign object within the cell. Such changes would be rather trivial, and written off as reflecting various degrees of damage, were it not for the observations that numerous combinations of resting and stimulus-related potential changes can occur. Since the sorting out of these relationships may be informative about the cell's transducer function, an elaboration of possible mechanisms is warranted.

In order that plausible mechanisms for the lability phenomenon may be formulated, some theoretical considerations from another paper (Dallos, 1984) are repeated for the sake of clarity. A simple circuit model (Fig. 7) for cochlcar hair cclls which may prove useful in explaining the differences between $\mathrm{IHC}$ and $\mathrm{OHC}$ resting potentials (Dallos, 1983, 1984) assumes that either hair cell may be represented by resistances $R_{\mathrm{a}}$ and $R_{\mathrm{b}}$, symbolizing the apical (endolymphatic) and basolateral (perilymphatic) boundaires of the cells, and a battery $E_{1}$ which describes the electrochemical potential of the basolateral membrane. These series elements, three for $\mathrm{OHCs}$ and three for ${ }_{H C S}$, are paralleled by a branch containing a battery $E_{T}$ and resistor $R_{T}$, representing the remainder of the cochlear circuit. $E_{T}$ is equivalent to the DC endochlear potential. Parametric excitation of the circuit is assumed via stimulus control of $R_{\mathrm{a}}^{0}$ and $R_{\mathrm{a}}^{1}$. Specifically, $R_{\mathrm{a}}^{0}=R_{1}^{0}$ $+\Delta R^{0}$ and $R_{\mathrm{a}}^{1}=R_{1}^{1}+\Delta R^{1}$. We will symbolize fractional resistance changes as $y^{0}=\Delta R^{0} / R_{1}^{0}$ and $y^{\prime}=\Delta R^{\prime} / R_{1}^{\prime}$. Capacitative reactances are ignored (but see Dallos, 1984, for a more complete treatment). One can show from the circuit that the voltages analogous to the

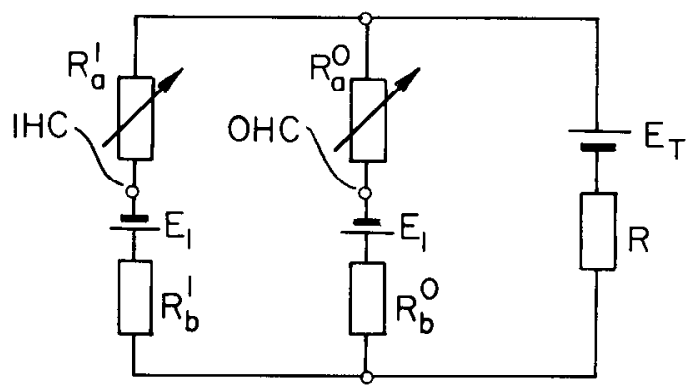

Figure 7. Circuit schematic diagram of one $\mathrm{OHC}$ and one $\mathrm{HC}$. The cells are represented, first, by the resistances of their basolateral (perilymphatic) surfaces, $R_{b}^{0}$ and $R_{b}^{1}$, which are assumed to be constant, and second, the resistances of the apical (endolymphatic) surfaces, $R_{a}^{0}$ and $R_{a}^{1}$, which are assumed to be variable and under control of the input stimulus. Finally, the batteries, $E_{1}$, represent the electrochemical potential of the basolateral membrane surface. The entire organ of Corti electrical circuit external to the hair cells is represented by its equivalent electrical resistance, $R$, and a battery, $E_{T_{1}}$ equivalent to the $\mathrm{DC}$ resting potential measurable in the scala media (endocochlear potential). This circuit and the assumptions governing its use are described by Dallos (1983). 
resting intracellular potentials $\left(E_{0}\right.$ and $\left.E_{1}\right)$ depend on the ratios of basolateral and apical resting resistances, $R_{\mathrm{b}} / R_{1}$. These ratios were named "shape factors" and they were expressed for OHCs: $\alpha=$ $R_{\mathrm{b}}^{0} / R_{1}^{0}$ and for IHCs: $\beta=R_{\mathrm{b}}^{\mathrm{b}} / R_{1}^{\mathrm{l}}$. The resting $\mathrm{DC}$ potentials $E_{0}$ and $E_{\mathbf{1}}$ may be expressed as follows (Dallos, 1983)

$$
E_{0} \approx \frac{\alpha E_{T}-E_{1}}{1+\alpha} \text { and } E_{1} \approx \frac{\beta E_{T}-E_{1}}{1+\beta}
$$

In the original modeling work it was assumed that for "good" $\mathrm{HCS}$ and $\mathrm{OHCs}$ the membrane potentials are -40 and $-70 \mathrm{mV}$, respectively. With $E_{1}=80$ and $E_{T}=70 \mathrm{mV}$, these numbers yield $\alpha=0.07$ and $\beta=0.36$. As equation 1 indicates, $E_{0}$ or $E_{1}$ may be influenced by variations in $E_{1}$ or either $\alpha$ or $\beta$. Assuming first that $\alpha=0.07$ and $\beta=0.36$, with $E_{T}=70$, all remaining constant while $E_{1}$ is variable, one can plot the theoretical change in $E_{0}$ and $E_{1}$. In Figure 8 these patterns are depicted. One notes that the fractional change in membrane resting potential is a linear function of the fractional change in $E_{1}$ : as $E_{1}$ increases, the cell hyperpolarizes, and it depolarizes with a decrease in $E_{1}$. It is theoretically possible for the resting potential to become positive if $E_{1}$ drops below the value $\alpha E_{T}$ or $\beta \mathrm{E}_{T}$, respectively. The dependence of the resting potential upon the shape factor is shown in Figure 9. It is seen that if the shape factor falls below its normal value, the resting membrane potential increases and tends towards an asymptote as the shape factor approaches zero. With an increase in the shape factor, the membrane potential falls (depolarization) and may reverse polarity. At very large values of the shape factor an asymptotic behavior of the membrane potential occurs, but here the asymptote represents a positive intracellular potential.

During the experiments both increases and decreases in resting membrane potentials were seen. Since variations in either $E_{1}$ or the shape factors can yield changes in both directions, one cannot

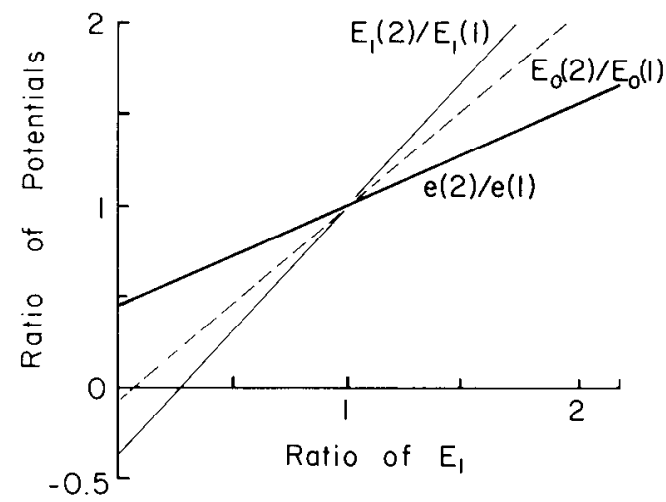

Figure 8. Theoretical dependence of the ratio of membrane potentials $\left(E_{1}\right.$ and $E_{0}$ ) and receptor potentials $(e)$ upon changes in the electrochemical driving force $\left(E_{1}\right)$. The straight lines can be derived from equations 1 and 2 .

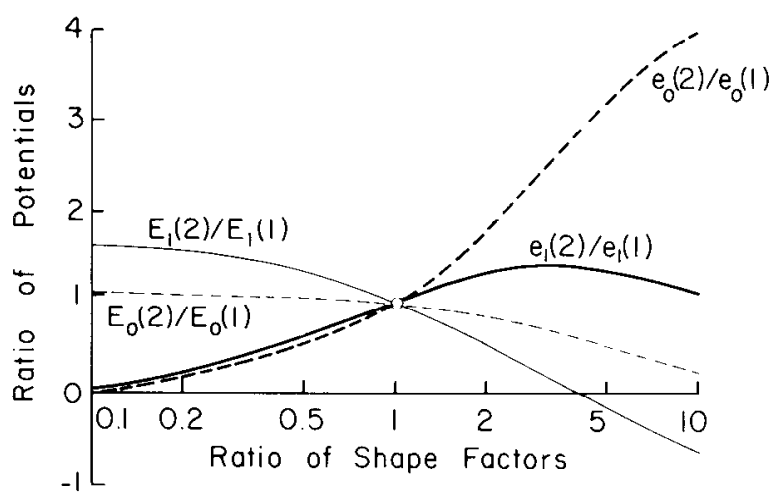

Figure 9. Theoretical dependence of the ratio of membrane potentials $\left(E_{1}\right.$ and $\left.E_{0}\right)$ and receptor potentials $\left(e_{1}\right.$ and $\left.e_{0}\right)$ upon changes in shape factors. The functions can be derived from equations 1 and 2 easily assign a mechanism to a given experimental finding. To better dissect the observed phenomena, we need to consider how receptor potential changes are affected by variations in $E_{1}$ and in the shape factor.

Stimulus-related potentials. When reactive circuit elements are ignored, the simplified electrical model of hair cell operation (Dallos, 1983) provides a formulation for receptor potentials as a function of apical resistance change. For IHCs and $\mathrm{OHCs}$ the linear approximation of the potential change is expressed as follows:

$$
e_{1} \approx \frac{\beta\left(E_{1}+E_{T}\right) y^{1}}{(1+\beta)^{2}} \text { and } e_{0} \approx \frac{\alpha\left(E_{1}+E_{T}\right) y^{0}}{(1+\alpha)^{2}}
$$

It is seen that for a given fractional resistance change $(y)$, the circuit voltage which is equivalent to the receptor potential is a function of the shapc factors and the electrochemical potential $E_{1}$ (the scala media resting potential $E_{T}$ is assumed not to be a variable). The electrode penetration may influence either the shape factor of $E_{1}$ or both. First, consider the effect of $E_{1}$ change. In Figure 8 the resulting graphs are provided, again with the numerical substitution of $E_{T}=$ $70 \mathrm{mV}$. The fractional change of the response is a linear function of the fractional change in $E_{1}$; moreover, the same plot represents the relationship for both the $\mathrm{OHC}$ and the $\mathrm{HC}$. The plot indicates that hyperpolarization increases, whereas depolarization decreases the stimulus-related potential.

If it is assumed that $E_{1}$ remains constant and the clectrodo penetration affects the shape factor, then one can obtain the fractional change in the response with variation in $\alpha$ or $\beta$ (numerical values are again assumed to be $E_{1}=80 \mathrm{mV}, E_{T}=70 \mathrm{mV}, \alpha(1)=$ $0.07, \beta(1)=0.36$ ). The relevant plots are included in Figure 9. It is noted that when the shape factors become smaller, the response magnitude tends toward zero. In contrast, as the shape factor increases, the response first grows to reach a maximum, then decreases toward zero for very large values of the shape factor. There are several interesting features of this behavior. For $\mathrm{OHCs}$ at least, there is a possibility for substantial enhancement of tho receptor potential with a rise in $\alpha$. A potential confusion may arise as to whether a given behavior is caused by increase or decrease in the shape factor, since either a large decrease of a large increase results in the diminution of the response. Fortunately, a comparison with concomitant changes in resting potential reveals that the response decrease that would be caused by the growth of the shape factor would be accompanied by a reversal of the polarity of the resting potential. Thus, if our scheme is correct, the potential confusion is easily averted. In fact, it is the relationship between changes in resting and receptor potentials that proves most valuable in attempting to assign mechanisms that underlie a variety of observed behaviors. Let us examine these relationships.

Resting versus receptor potentials. It may be best to organize the comparisons between resting and membrane potential changes and their plausible explanations in the graphical format of Figure 10. On the left side the experimental observations are summarized; the right side shows permissible outcomes from the previously discussed model predictions. The symmetrical figures show the nine possible relationships between resting potential $(E)$ and receptor potential $(e)$, when these increase, decrease, or remain steady. Hatched boxes on the left side of Figure 10 indicate that no experimental observation corresponds to the given condition, whereas references to figures in this paper, inserted in other boxes, suggest a possible experimental situation. The boxes of the right side, similarly organized according to the direction of change in $E$ and $e$, are filled with the aid of data from Figures 8 and 9 . Assuming that a reasonable range of change in $\alpha$ and $\beta$ is from 0.1 to 10 times normal, one simply reads from the graphs what behavior corresponds to a given mechanism. Thus, we note that the model does not allow for changes in the receptor potential $(e)$ without a concomitant change in the membrane potential $(E)$. Boxes that correspond to such "nonpermissible" behavior are cross-hatched. It is further seen that simultaneous increase or decrease in both $E$ and 

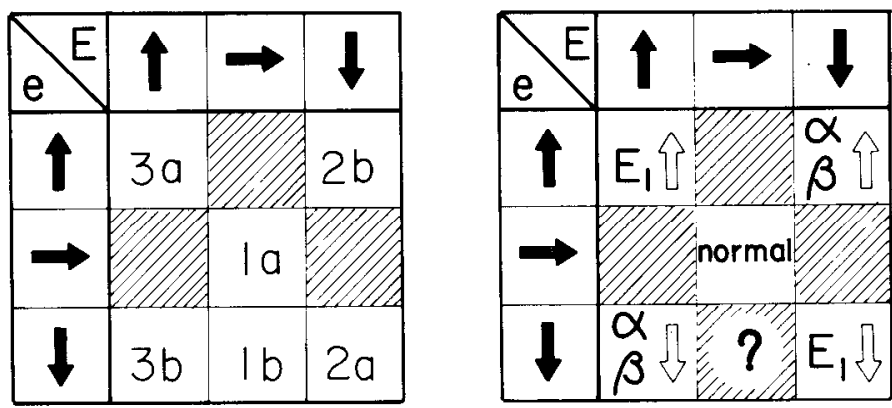

Figure 10. Relationship between direction of change in membrane po tential $(E)$ and receptor potential $(e)$. Directions are indicated by the heavy arrows: up, steady, (horizontal arrow), and down. Left, Experimental observations. Hatched squares indicate no data available that would correspond to the given relation bctwcon $E$ and $e$. Numbers in other boxes indicate the figure numbers in this paper in which the particular condition ( $E-e$ pair) is depicted. Right, Theoretical relationship between changes in membrane potential and receptor poterilial. From equalions 1 and 2 and from Figures 8 and 9 one notes that certain pairings are not permitted. These are cross hatched. Permissible possibilities are shown in the other boxes, along with the likely cause. Thus, $t_{1}$ or the shape factors may increase or decrease (open arrows), causing the associated changes in $E$ and $e$. The simple formulations of equations 1 and 2 do not readily explain the experimental findings of the bottom center box; thus, this condition is denoted by a question mark.

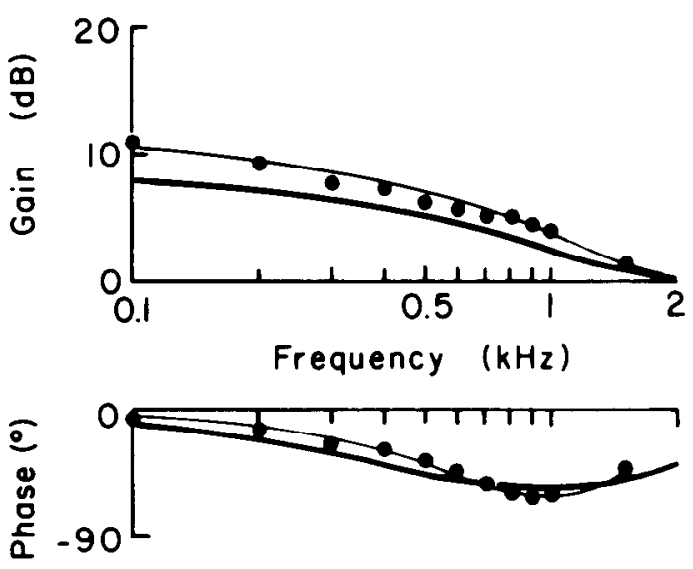

Figure 11. Magnitude and phase changes (relative to the organ of Corti response) of the $A C$ receptor potential during a decrease in the membrane potential of the receptor cell shown in Figure $2 b$. Data are shown by the solid circles connected with the thin line. The thick line indicates the theoretical changes as computed with the aid of the formula given in Footnote 3 . It is seen that both magnitude and phase changes are frequency depend. ent. In this case a greater magnitude change obtains at low frequencies, whereas the phase change is maximal around the BF. The magnitude change indicated in Figure 2 refers to that seen at BF, i.e., $+5 \mathrm{~dB}$.

$e$ is possible if electrode penetration affects $E_{1}$, the electrochemical driving voltage (the up-up and down-down boxes). Finally, $E$ and $e$ move in opposite directions if the cause is a change in shape factor (up-down and down-up boxes). Of course, the steady-steady situation is a trivial one. In comparing the two arrays of Figure 10, there seems to be a satisfying correspondence between not observed and "not permissible" pairings. The only anomaly corresponds to the case in which the response was seen decreasing while the membrane potential remained steady (example: Fig. 1b). This case is noted by a question mark on the right side. Reduction in response size without a change in membrane potential is a puzzling observation. The simple hair cell circuit model does not provide an explanation for this set of tindings. One plausible cause for the observations is that the recording electrode somehow interferes with the cell's transducer mechanism.

It is probably not necessary to emphasize that the above consid- erations embodied the obvious simplification of assuming a change in either $E_{1}$ or the shape factor. It is likely that membrane changes due to the presence of the recording electrode would yield simultaneous alterations in both parameters. If so, the eventual consequence depends on the interplay of the effects produced by the degree and direction of change in $E_{1}$ and, say, $\beta$. Intuitive pairings would tie increasing $E_{1}$ with increasing shape factor (sealing of the cell membrane) and, in contrast, a membrane that becomes more "leaky" may well yield decrease in $E_{1}$ and in shape factor. In the former case an increase in response is expected from both factors, but the fate of the membrane potential is uncertain. The converse case yields decrease in responses, again with indeterminate outcome to the membrane potential.

It is at least conceivable, albeit not very likely, that opposing effects of $E_{1}$ and $\beta$ are such that the membrane potential may remain steady, whereas the response undergoes a decrease. This could explain the experimental observation, and its lack of theoretical correlate, in the bottom row center box of Figure 10.

Lability of tuning. Certain combinations of membrane and receptor potential changes could be explained in terms of parameter variations in a simple electrical circuit model of hair cells. We have seen with the aid of Figure 10 that if either the electrochemical driving force $\left(E_{1}\right)$ or the shape factors $(\alpha$ or $\beta$ ) are altered as a consequence of electrode entry into the cell, then the direction of change of both membrane and receptor potential may be predicted. in a similar vein one can consider the effect of these parameter changes upon the filter characteristics of the cell. It is easy to show that the only expected effect upon luning is a consequence of alterations in $\alpha$ or $\beta$. Specifically, the membrane time constant $(\tau)$ changes as the shape factors are altered but remains unchanged if $E_{1}$ is varied. For $\mathrm{HCs}$ the time constant changes from its resting value $\tau^{\prime}(1)$ to a new value $\tau^{\prime}(2)$ with a shift in shape factor from $\beta(1)$ to $\beta(2)$ in the following manner:

$$
\tau^{\prime}(2) / \tau^{\prime}(1)=\beta(2)[1+\beta(1)] / \beta(1)[1+\beta(2)]
$$

The corner frequency of the membrane filter is inversely related to the time constant, $f=2 \pi \tau$. As the shape factor decreases the time constant also decreases, which is equivalent to saying that the membrane filter's cutoff frequency increases. This change is best visualized by noting that this condition corresponds to the basolateral membrane becoming less resistant, more "leaky." If the corner frequency goes to higher values, presumably as a consequence of the decrease in $\beta$, then one expects to see a decrease in the low frequency response magnitude and a concomitant expansion of the region of low frequency phase lead to higher frequencies. Conversely, an increase in $\beta$ should yield a reduction in the low frequency phase lead along with an increase in response magnitude. ${ }^{3}$ For

\footnotetext{
${ }^{3}$ Actually, the situation is more complex. The ratio of $\mathrm{IHC}$ response to that in the organ of Corti may be expressed as follows (Dallos, 1984):$$
\frac{e_{1}}{e_{o C}} \approx \frac{\text { const. }}{1+\beta} \frac{j \omega}{\frac{1+\beta}{T \beta}+j \omega}
$$

where $T$ is a hybrid time constant, combining apical cell membrane resistance with total membrane capacitance. After substituting a reasonable numerical value for $T$, one may compute magnitude and phase changes of the above ratio as a joint function of $\beta$ and $\omega$. At any given frequency, the magnitude function possesses a maximum at a $\beta$ value that varies between 0.1 and 1.0. The originally estimated $\beta=0.36$ is at the maximum around the BF. This means that, if upon penetration of the cell $\beta$ is low and then subsequently increases, then the response magnitude is expected to increase throughout the frequency range. In contrast, starting out with normal $\beta$ and suffering a decrease in this value during penetration would yield a drop in response Ambiguous situations might arise if $\beta$ were to increase from its normal value. This would produce either increase or decrease in the response, depending on measuring frequency. The change in phase is simpler. A decrease in $\beta$ produces an increased lead at any frequency and vice versa. The degree of change, however, is frequency dependent; at low frequencies the change is minimal, and higher frequencies generate more phase change.
} 
$\mathrm{OHCs}$ a change in $\alpha$ simply yields a broadening or narrowing of the low-pass filter. When the shape factor increascs, the filter narrows and the magnitude increases within the passband, whereas a decreasing $\alpha$ yields broader filter and smaller response.

A rereading of the appropriate portion under "Results" and reference to Figure 10 indicates that tuning changes should accompany those experimental conditions in which one may surmise changes in shape factors. Thus, membrane potential-receptor potential variations that can be attributed to changes in $E_{1}$ are not characterized by tuning changes. In contrast, we noted that a condition signified by increasing membrane potential and decreasing response is also the hallmark of broadened tuning (Fig. 4). Such a condition was previously identified by a decrease in shape factor. The above argument indicates that filter changes seen are consistent with this interpretation of the cause. Similarly, we have identified situations in which membrane potential decreased in time and response magnitude increased. In Figure 11 magnitude and phase changes, corresponding to the membrane potential decrease depicted in Figure $2 b$, are presented. It is seen that there is a marked increase in magnitude at low frequencies and the gain is diminishing toward the high frequencies. The numerical value of $5 \mathrm{~dB}$ that was assigned in Figure $2 b$ obtains at the BF. I.ow frequency phase change is minimal, but as the membrane potential decreases, the result is a decreased lead toward high frequencies. This is, of course, manifested as an increasing phase lag as the phase of the later condition is referred to that seen in the early situation. Just to provide a measure of the success of the circuit model in predicting changes in tuning, it was arbitrarily assumed that $\beta$ changes from an early value of 0.1 to a later value of 0.36 . From the formula given in Footnote 3 one can obtain the change in magnitude and phase as a function of frequency, and these plots are also included in Figure 11. The correspondence between experimental data and prediction is acceptable, particularly the similar frequency-dependent configurations. This agreement suggests that, in this case, time-dependent alterations in cell function may be due to variation in shape factor.

The extreme broadening of the filter, along with a decrease in response magnitude to that seen in the extracellular space, which is depicted in Figure 4, can be interpreted as the diminishing of the shape factor toward zero. This may be simply a formalistic expression of very low basolateral membrane resistance due to a peculiar injury process. Specifically, it is conceivable that injury permits the entry of extracellular $\mathrm{Ca}$ and the activation of Ca-dependent $\mathrm{K}$ channels. The high $K$ flux would move the membrane toward the $K$ equilibrium potential and, at the same time, the lowered membrane resistance would shunt the intracellular receptor potential. Apparently, A. J. Hudspeth (private communication) also observed in saccular hair cells this peculiar increase in membrane potential and a decrease in receptor potential. The demonstration (Lewis and Hudspeth, 1983) of the existence of Ca-dependent $\mathrm{K}$ channels in hair cell lateral membranes makes the above explanation of the phenomenon plausible. It is also conceivable that the process would force the possibly electrogenic Na-K pump into higher than normal activity and thus further hyperpolarize the cell membrane.
An alternative view may be that the extreme decrease in shape factor that could producc the observed phenomenon is not due to the diminishing of the basolateral resistance, but to a radical increase in the apical resistance. Perhaps if the transducer channels became largely nonfunctional due to injury, then a formalistic representation of this is an increase in $R_{1}$, yielding a decrease in $\alpha$. In this situation the cell's electrical behavior mimics that of an organ of Corti sup. porting cell. The linearization of the response (symmetrical waveforms not containing a significant DC response component) seen in these cases (Fig. 6) could then be a result of the interference with the transducer channel.

\section{References}

Brown, M. C., A. L. Nuttall, R. I. Masta, and M. Lawrence (1983) Cochlear inner hair cells: Effects of transient asphyxia on intracellular potentials. Hear. Res. 9: 131-144.

Crawford, A. C., and R. Fettiplace (1981) Non-linearities in the responses of turtle hair cells. J. Physiol. (Lond.) 315: 317-338.

Dallos, P. (1983) Some electrical circuit properties of the organ of Corti. I. Analysis without reactive elements. Hear. Res. 12: 89-119.

Dallos, P. (1984) Some electrical circuit properties of the organ of Corti. II. Analysis including reactive elements. Hear. Res. 14: 281-291.

Dallos, P. (1985) Response characteristics of mammalian cochlear hair cells. J. Neurosci. 5: 1591-1608.

Fettiplace, R., and A. C. Crawford (1978) The coding of sound pressure and frequency in cochlear hair cells of the terrapin. Proc. R. Soc. Lond. (Biol.) 203: 209-218.

Flock, А., M. Jørgensen, and I. Russell (1973) The physiology of individual hair cells and their synapses. In Basic Mechanisms in Hcaring, A. Møllcr, ed., pp. 273-301, Academic Press, Inc., New York.

Goodman, D. A., R. L. Smith, and S. C. Chamberlain (1982) Intracellular and extracellular responses in the organ of Corti of the gerbil. Hear. Res. 7: $161-179$.

Harris, G. G., L. Frishkopf, and A. Flock (1970) Receptor potentials from hair cells of the lateral line. Science 167: 76-79.

Hudspeth, A. J. (1983) Mechanoelectrical transduction by hair cells in the acousticolateralis system. Annu. Rev. Neurosci. 6: 187-215

Hudspeth, A. J., and D. P. Corey (1977) Sensitivity, polarity, and conductance change in the response of vertebrate hair cells to controlled mechanical stimuli. Proc. Natl. Acad. Sci. U. S. A. 74: 2407-2411.

Lewis, R. S., and A. J. Hudspeth (1983) Voltage and ion-dependent conductances in solitary vertebrate hair cells. Nature 304: 538-541.

Nuttall, A. L., M. C. Brown, R. I. Masta, and M. Lawrence (1981) Inner hair cell responses to velocity of basilar membrane motion in the guinea pig. Brain Res. 211: 171-174.

Russell, I. J. (1983) Origin of the receptor potential in inner hair cells of the mammalian cochlea-Evidence for Davis' theory. Nature 301: 334-336.

Russell, I. J., and J. F. Ashmore (1983) Inner hair cell receptor potentials investigated during transient asphyxia: A model for hair cell coupling. In Hearing-Physiological Bases and Psychophysics, R. Klinke and R. Hartmann, eds., pp. 10-15, Springer-Verlag, Berlin.

Russell, I. J., and P. M. Sellick (1978) Intracellular studies of hair cells in the guinea pig cochlea. J. Physiol. (Lond.) 284: 261-290.

Russell, I. J., and P. M. Sellick (1983) Low-frequency characteristics of intracellularly recorded receptor-potentials in guinea-pig cochlear hair cells. J. Physiol. (Lond.) 338: 179-206.

Weiss, T. F. (1984) Relation of receptor potentials of cochlear hair cells to spike discharges of cochlear neurons. Annu. Rev. Physiol. 46: 247-259. 